\title{
]jfis
}

\section{A Novel Infrastructure Design of Industrial Autonomous System}

\author{
Quang Thinh Truong ${ }^{1}$, Ha Quang Thinh $\mathrm{Ngo}^{1 *}$, Thanh Phuong Nguyen ${ }^{2}$, Hung \\ Nguyen $^{2}$, and Won-Ho Kim ${ }^{3}$ \\ ${ }^{1}$ Graduate Student, ${ }^{1 *}$ Department of Mechatronics Engineering, Ho Chi Minh City University of Technology \\ (VNU-HCM), HCMC, Vietnam \\ ${ }^{2}$ Institute of Engineering, Ho Chi Minh City University of Technology, HCMC, Vietnam \\ ${ }^{3}$ Major of Robot · Automation Engineering, Dong-Eui University, Busan, Korea
}

Received: Mar. 12019

Revised : Jun. 14, 2019

Accepted: Jun. 23, 2019

Correspondence to: Ha Quang Thinh Ngo (nhqthinh@hcmut.edu.vn)

(T)The Korean Institute of Intelligent Systems

CCThis is an Open Access article distributed under the terms of the Creative Commons Attribution Non-Commercial License (http://creativecommons.org/licenses/ by-nc/3.0// which permits unrestricted noncommercial use, distribution, and reproduction in any medium, provided the original work is properly cited.

\begin{abstract}
In this era, the integrated technologies in a unit bring huge benefits for customers. In this paper, a novel design of structure for autonomous system is illustrated. It is superior by combining the specifications of various types in commercial market. As a result, numerous advantages of systems are converged into unified unit. The design of autonomous platform consists of hardware components and infrastructure. Later, the modeling of autonomous system is simulated on computer to fit with proposed design. The implementation of control scheme into model is to drive autonomous system to complete mission. From simulation results, it can be seen that the proposed strategy is feasible, effective and capable in reality.
\end{abstract}

Keywords: Robotics, Control topology, Industrial automation, Forklift, Automated vehicle

\section{Introduction}

In every production site, the occupations for loading, unloading and transporting of cargoes or materials are always to use much labor resource. These jobs are not complex, repeated actions and lack of intelligence. Sometimes, worker feels boring or emotion-based productivity. The managers worry about their work-force in each quarter of year. While the high operation cost reduces the competitive ability of company, the faster processing of bill will meet customer satisfaction. The automatic solution should be considered in these situations. The autonomous system is automated transporters that have been developed to support the industrial enterprises gain high productivity with minimum cost. The automated solution could be adjusted size of system due to needs, effective execution, adaptable producing schedule or scalable model. In last decades, there are large numbers of accomplished investigation to use autonomous system in industry. Most of them focus on designing automated vehicle which plays an important role in autonomous system. Depending on each specific application, the core of system could be divided into many kinds of sub-vehicles.

For automated warehouse management, several famous models have been declared firstly. In [1, 2], the area of storehouse is divided into many sections such as charging station, delivering station, importing station or location of storing cargo. The man operators are at delivering station or importing station while autonomous vehicles carry shelves inside. In large distribution center, this model is more productive than traditional methods. The path 
planning for these applications in presence of obstacles could be $\mathrm{A}^{*}$ based on vision [3, 4] or Dijkastra [5] shortest path algorithm. However, this type is appropriate for small freights in shelves, logistics system and lacks of automatic feeder actuators.

In the other of load transportation applications which involve heavy weight or feeder component, more than one shelf, it is usually to extend the functionalities of traditional autonomous system. The towing-vehicle such as truck or forklift [6] gains more advantages. In [7], the module-based system permits to modify the number of robots and pallets to lessen operating cost and consuming time. The software architecture is flexible to add more modules while it still keeps the system's structure. Researchers in [8, 9] present a robotic forklift for intelligent warehouse. Three algorithms are implemented in forklift, routing algorithm, local path planning algorithm and an auto-localization algorithm. However, this research can meet serious problems in real time control due to the order of path planning steps before sending to robot. Also, it has not validated in experimental multi robotic forklifts scenario.

Recently, the new trend of integrated autonomous vehicle occurs in most of industries. The automated devices work in logistics applications while it still maintains the capable operation in material delivering applications. To gratify the requirements of customers, the developers must integrate various functions into one unit though the benefits of physical dimension, light weight and cheap price are kept. In this paper, the novel design of industrial autonomous system with multifunction is investigated. The system parameters such as size, load capacity, operation mode, communication or tracking method ensure the usage in various fields. Section 2 consists of the description of system and specifications. The hardware structure of autonomous system is illustrated in Section 3. The content of modeling and simulation is described in following part, Section 4. Several results of proposed design in Section 5 are demonstrated to verify in industries. Finally, conclusions and future works to develop are carried out.

\section{System Parameters and Specifications}

In this system, it is necessary to provide a powerful management to process all of peripheral devices. In Figure 1, the connection diagram between micro-processor and other components is illustrated. The high-performance ARM Cortex M4 32-bit operating at max frequency of $168 \mathrm{MHz}$ is considered as core of CPU module. This embedded processor features a floating-

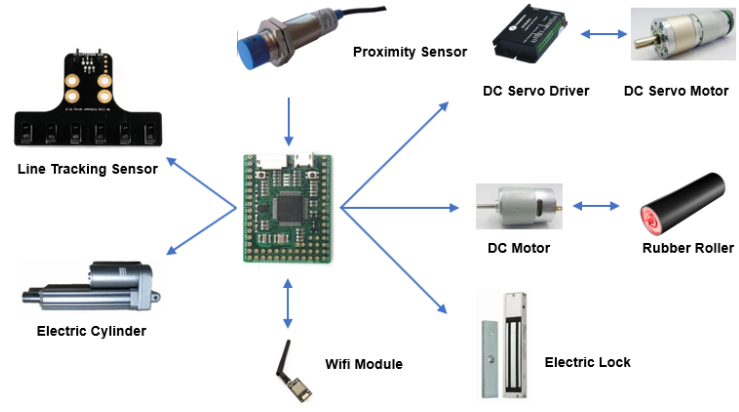

Figure 1. Block diagram of control system in proposed system.

Table 1. Parameters and specifications of system

\begin{tabular}{lc}
\hline \multicolumn{1}{c}{ Items } & Descriptions \\
\hline Physical dimension & $650 \times 420 \times 350 \mathrm{~mm}$ \\
Wheels & 4 (2 driving wheels, 2 castor wheels $)$ \\
Max speed & $0.5 \mathrm{~m} / \mathrm{s}$ \\
Driving mode & Differential drive \\
MCU & ARM STM32F405 \\
Power & 2 battery 12 VDC-48Ah \\
Tracking method & Line follower or magnetic sensor \\
Sensors & Loadcell, proximity, current \\
Carrying mode & Lift-up, trailer \\
\hline
\end{tabular}

point unit (FPU) single precision which supports all singleprecision data processing instructions. Some key specifications such as 1 Mbyte flash, 192 kbytes SRAM, 3 channels A/D 12 bit, 2 channels D/A 12-bit offer a wide range of applications. The two DC servo motors with power $60 \mathrm{~W}$ and max speed 70 rpm are able to drive whole system. Due to the different speeds among servo motors, the autonomous vehicle can move easily and conveniently. There are several ways to track the reference trajectory. Generally speaking, using line following sensor or magnetic tracking sensor are two popular methods. To enhance the competition of this system in market, line following sensor becomes the best solution to implement. The micro-controller can control electric cylinder to lift up or down cargo with max load $200 \mathrm{~kg}$. Moreover, this robot is equipped with electric lock which is capable to hold a powerful electromagnetic force to drive followers. Additionally, the human operator must stand at start point and target location to pick up freight. The operating cost will be increased in traditional system. In this design, mobile robot will be run from beginning to ending steps lonely. Thanks to roller actuators, the automated ability of robot can be improved. 


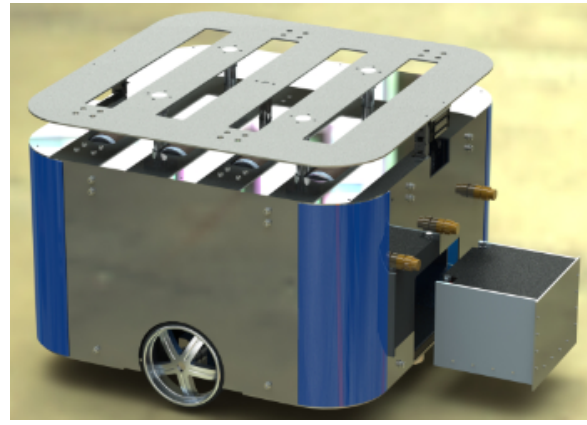

Figure 2. Overview of proposed autonomous vehicle.

The system coefficients and operating information are listed as Table 1 . The physical size is large enough to carry various loads while it still maintains the flexible execution. In some cases, the suitable height of this vehicle helps its move underneath the shelves. Hence, it can work anywhere in map regarding to dimension. In industry, the working route consists of two kinds: uni-direction and bi-direction. To deal with numerous maps, in this work, the autonomous vehicle should move in forward and reverse path. Furthermore, in some specific situations such as material transportation in clothing or textile industry, robot extricates one or more shelves. As a result, the power of motor and tires of wheels are studied to optimize selection.

\section{Description of Proposed Design}

In Figure 2, the whole vehicle is shown to overview the structure. Basically, robot can be divided into four sections: base layer, middle layer, feeder layer and lifting layer. Each layer plays own role such that base layer is strong enough to bear the body mass and load. The linear slider leans on middle layer to orientate vertical direction while the feeder layer hangs rubber rollers to provide cargo automatically. Top layer or lifting layer is elevated by electric cylinder and directly contacts to cargo.

The driving mechanism consists of two driving wheels on side, castor-type wheels in front and back. In Figure 3, this structure avoids the slipping phenomenon, guarantees flexible motion and all wheels in contact with a certain surface. Two powerful motors are connected with shafts of wheels via gear boxes. The directional movement depends on the difference between speed of left wheel and right wheel. The following path sensors are located at center of bottom layer. In both sides of forward and backward, it provides bi-directional driving ability in map. Besides, RFID reader module on this layer helps to navigate via tags which are placed at each intersection. By

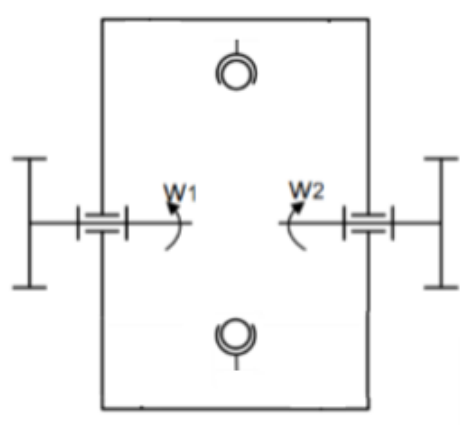

(a)

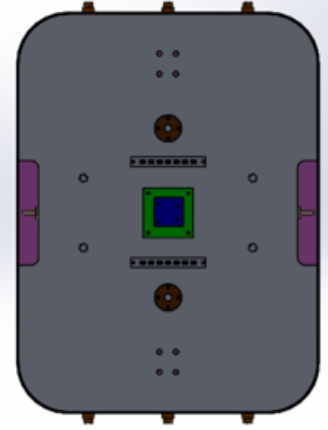

(b)
Figure 3. Driving mechanism on bottom layer: (a) theoretical design and (b) 3D model.

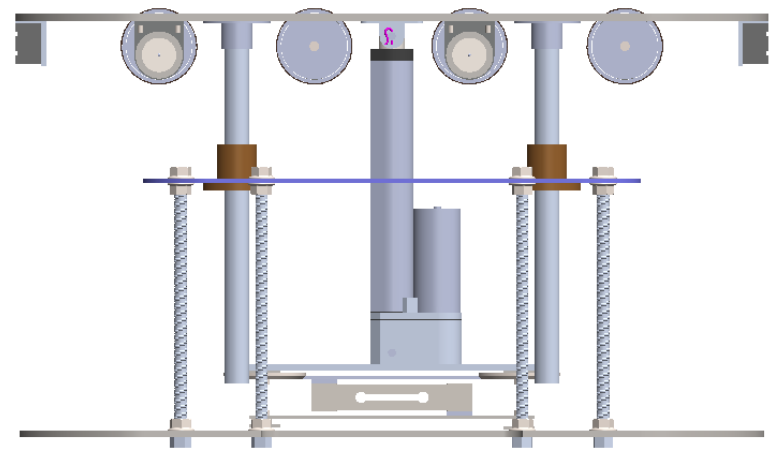

Figure 4. Inside architecture of loading and feeding mechanism.

reaching a crossroad, vehicle reads identification numbers of tags and returns to host computer. Therefore, server could track the following path and generate the proper trajectory.

The structure of ground vehicle is demonstrated in Figure 4. The electric cylinder mainly provides lifting force to elevate freight while four slider actuators linearly preserve vertical movement. Under the electric piston, there is a load-cell to evaluate the weight level of goods. As a result, the automated vehicle is able to carry out proper control signal smoothly. Furthermore, it is enhanced the advanced functions via the feeding mechanism. Whenever mobile vehicle stops at destination, without supporters, it automatically delivers freight to storage. In both of head and tail, the electromagnets are attached to lead the behind carriages. In this case, the overall vehicle-load plays a role as tractor-trailer system.

\section{Modeling of Proposed System}

To figure the performance of proposed system, the kinematic modeling in Figure 5 describes the geometrical relationship of parameters. We consider that geometric center $\mathrm{C}$ and the 


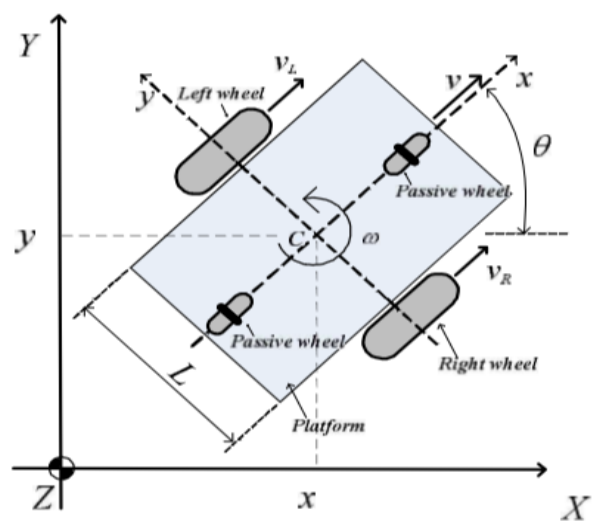

Figure 5. Kinematic relationship of vehicle model.

center of gravity are coincided. $q=[x, y, \theta]^{T}$ symbolize the coordination of vehicle in workspace.

where

- $\theta:$ an angle between vertical axially length and $\mathrm{Ox}_{0}$.

- L: distance between wheels.

- $\theta_{P}:$ an angle between $\mathrm{Ox}_{0}$ and tangent.

- $\mathrm{x}_{P}, \mathrm{y}_{P}$ : desired coordinate of CoG.

- $\mathrm{x}_{C}, \mathrm{y}_{C}$ : coordinate of CoG.

The kinematic equation of autonomous vehicle at center point $\mathrm{C}, \mathrm{CoG}$ and point $\mathrm{P}$ of trajectory are illustrated correspondingly.

$$
\begin{aligned}
& {\left[\begin{array}{c}
\dot{x}_{M} \\
\dot{y}_{M} \\
\dot{\theta}
\end{array}\right]=\left[\begin{array}{cc}
\cos \theta & 0 \\
\sin \theta & 0 \\
0 & 1
\end{array}\right]\left[\begin{array}{c}
v \\
w
\end{array}\right],} \\
& \left\{\begin{array}{l}
\dot{x}_{C}=\dot{x}-a \dot{\theta} \sin \theta, \\
\dot{y}_{C}=\dot{y}+a \dot{\theta} \cos \theta, \\
\dot{\theta}_{C}=\dot{\theta},
\end{array}\right. \\
& {\left[\begin{array}{c}
\dot{x}_{P} \\
\dot{y}_{P} \\
\dot{\theta}_{P}
\end{array}\right]=\left[\begin{array}{cc}
\cos \theta_{P} & 0 \\
\sin \theta_{P} & 0 \\
0 & 1
\end{array}\right]\left[\begin{array}{c}
v_{P} \\
w_{P}
\end{array}\right],}
\end{aligned}
$$

where

- $v$ : linear velocity of mobile platform.

- $w$ : angular velocity of mobile platform.

- $v_{P}$ : desired linear velocity at reference point.

- $w_{P}$ : desired angular velocity at reference point.
- $\mathrm{d}$ : distance between center point of vehicle and middle point of driving wheels.

The error modeling $e=\left[\begin{array}{lll}e_{1} & e_{2} & e_{3}\end{array}\right]^{T}$ is defined as the alteration between $\mathrm{CoG}$ and desired point on reference path.

$$
\left[\begin{array}{c}
\dot{x}_{P} \\
\dot{y}_{P} \\
\dot{\theta}_{P}
\end{array}\right]=\left[\begin{array}{cc}
\cos \theta_{P} & 0 \\
\sin \theta_{P} & 0 \\
0 & 1
\end{array}\right]\left[\begin{array}{c}
v_{P} \\
w_{P}
\end{array}\right] \text {. }
$$

Take the first derivative order of Eq. (4), we have

$$
\left[\begin{array}{c}
\dot{e}_{1} \\
\dot{e}_{2} \\
\dot{e}_{3}
\end{array}\right]=\left[\begin{array}{c}
v_{P} \cos e_{3} \\
v_{P} \sin e_{3} \\
w_{P}
\end{array}\right]+\left[\begin{array}{cc}
-1 & e_{2} \\
0 & -e_{1}-d \\
0 & -1
\end{array}\right]\left[\begin{array}{c}
v \\
w
\end{array}\right] .
$$

Therefore, the stable controller is proposed for this design of autonomous vehicle.

$$
\left[\begin{array}{c}
v=v_{P} \cos e_{3}+k_{1}\left(e_{1}+a\right) \\
w=k_{2} e_{2} v_{P}+w_{P}+k_{3} v_{P} \sin e_{3}
\end{array}\right] .
$$

In the sense of Lyapunov stability, the function candidate $V$ should be positive definite and continuous in domain. At equilibrium point, the function $V$ must be zero. Consider a Lyapunov function candidate as following:

$$
V=\frac{1}{2}\left(e_{1}+d\right)^{2}+\frac{1}{2} e_{2}^{2}+\frac{1-\cos e_{3}}{k_{2}} .
$$

With condition $\forall k_{2}>0$, if $e=\left[\begin{array}{lll}e_{1} & e_{2} & e_{3}\end{array}\right]^{T}$ equals to zero, then $V=0$. Otherwise $e \neq 0$, then $V>0$.

With respect to time, take the derivative (7), we obtain

$$
\begin{aligned}
\dot{V}= & \left(e_{1}+d\right) \dot{e}_{1}+e_{2} \dot{e}_{2}+\frac{\dot{e}_{3} \sin e_{3}}{k_{2}}, \\
\dot{V}= & \left(e_{1}+d\right)\left(v_{P} \cos _{3}-v+e_{2} w\right) \\
& +e_{2}\left(v_{P} \operatorname{sine}_{3}-e_{1} w-w a\right)+\frac{\left(w_{P}-w\right) \sin e_{3}}{k_{2}}, \\
\dot{V}= & \left(e_{1}+d\right)\left(v_{P} \cos e_{3}-v\right) \\
& +\sin e_{3}\left(e_{2} v_{P}+\frac{w_{P}-w}{k_{2}}\right) .
\end{aligned}
$$

Using stabilizing controller from Eq. (6), we have

$$
\dot{V}=-k_{1}\left(e_{1}+d\right)^{2}-\frac{v_{R} k_{3}\left(\sin e_{3}\right)^{2}}{k_{2}}
$$

It can be seen clearly that $\dot{V} \leqslant 0, \forall k_{1}, k_{2}, k_{3}>0$. The tracking error converges to zero asymptotically when $v_{R}>0$. 


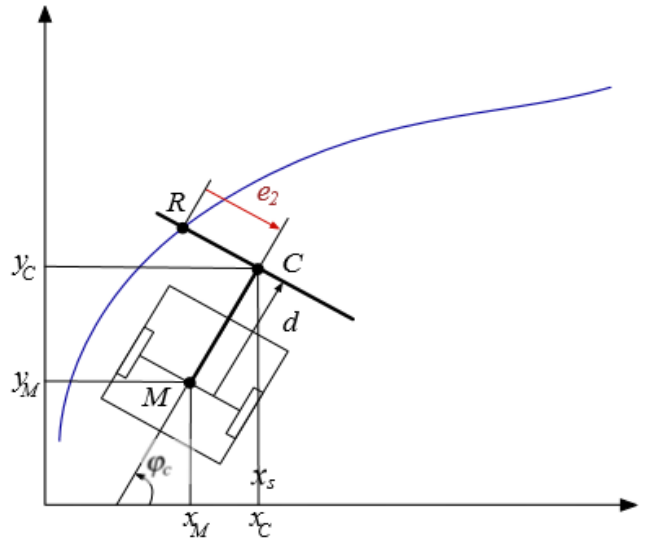

Figure 6. Description of tracking error $e_{2}$.

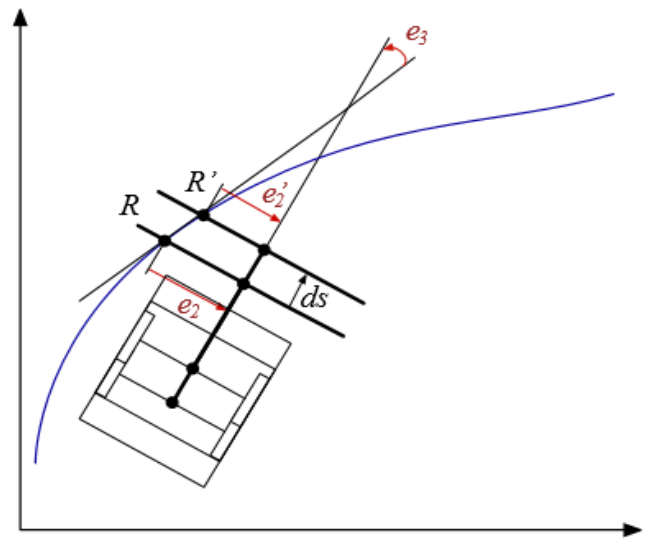

Figure 7. Description of tracking error $e_{3}$.

The velocity control input in (6) could guarantee the system is asymptotically stable as time goes to infinity when $\mathrm{d}$ is zero. If vehicle stays in parallel with reference trajectory, then $e_{1}=$ $e_{3}=0$ and $e_{2} \neq 0$. At that time, Eq. (6) of velocity control might not force $e_{2}$ to zero.

In practical model, it is necessary to gain the tracking error e to prove the effective drive. Consider that vehicle moves with reference speed, hence, $e_{1}=0$. The tracking sensors performs the $e_{2}$ error along normal direction with movement.

To compute the tracking error $e_{3}$, let vehicle move a small distance $d_{s}$. Assume that from point R to point R' on trajectory, it is nearly linear.

$$
e_{3}=\arctan \frac{e_{2}-e_{2}^{\prime}}{d_{S}}
$$

\section{Results of Research}

To validate the flexible motion and feasible control algorithm, the autonomous vehicle is trialed on working map as Figure 8.

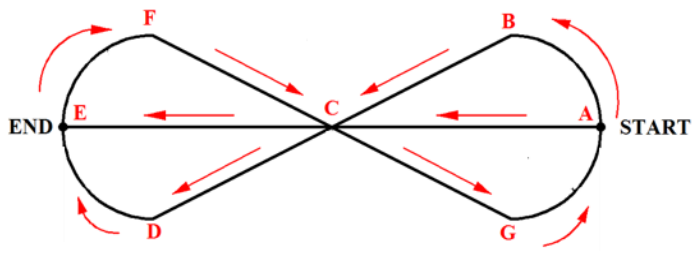

Figure 8. Tracking lines of working map.

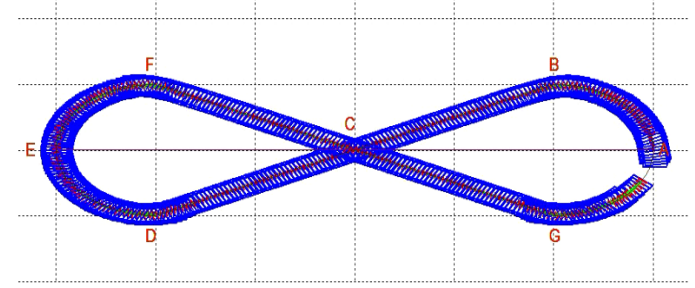

Figure 9. Simulations on overall working map.

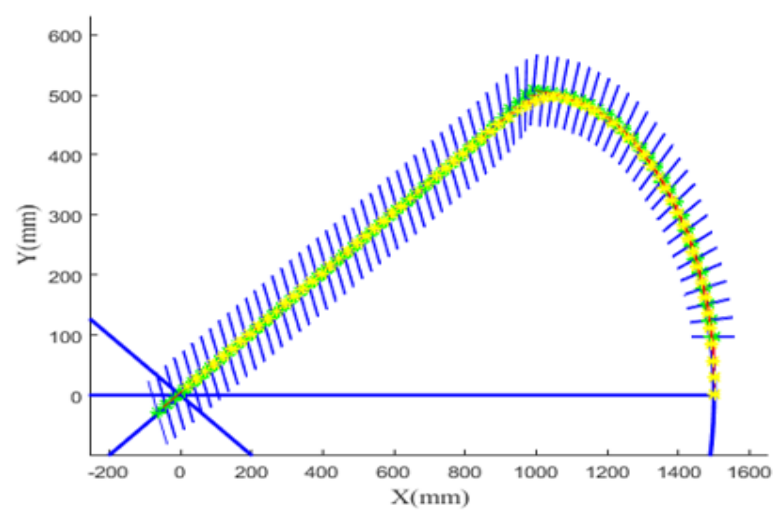

Figure 10. Simulation results for $\mathrm{ABC}$ section on working map.

There are two significant points, for example, start point and end point. However, the autonomous vehicle would follow one round from start point to evaluate performance on different situations. In reality, there are unique directional paths and bidirectional paths depending on applications. The proposed design is able to work in both cases. The tracking simulation of trajectory is shown in Figure 9 dividing into sub-sections, such as A-B-C, C-D-E-F, F-CG and C-G-A. In each section, vehicle tracks black line while it returns signals (tracking errors, speeds, current status and so on) to host computer. RFID tags locate at every point to navigate current coordination. The gains are selected based on experimental operator but satisfying positive value, $k_{1}=1.3, k_{2}=80, k_{3}=1.8$. As a result, the stable condition is ensured by Lyapunov approach.

In A-B-C section as Figure 10, at B point, there is a sudden changing direction to fetch bigger tracking errors. Though, from $\mathrm{A}$ to $\mathrm{B}$ or from $\mathrm{B}$ to $\mathrm{C}$, the errors fluctuate gradually as Figure 11. Whenever vehicle is in corner, speeds of vehicle in 

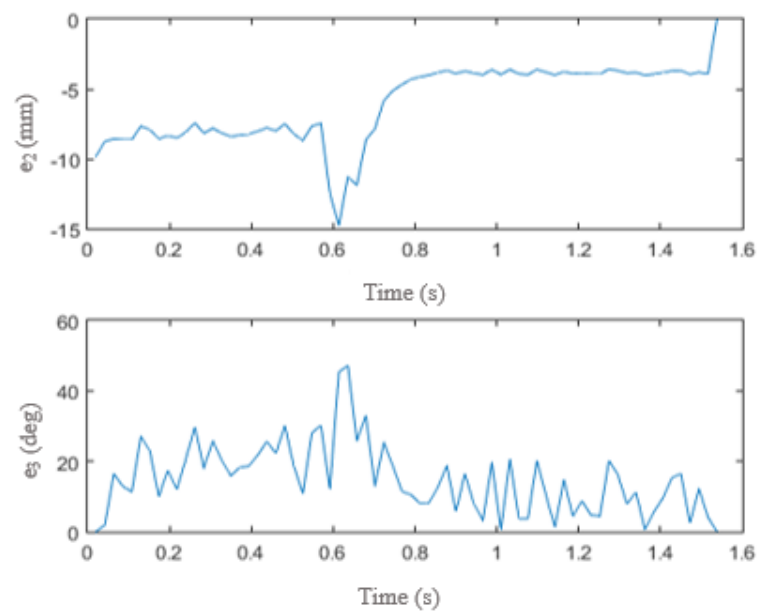

Figure 11. Results of tracking error for $\mathrm{ABC}$ section on working map.
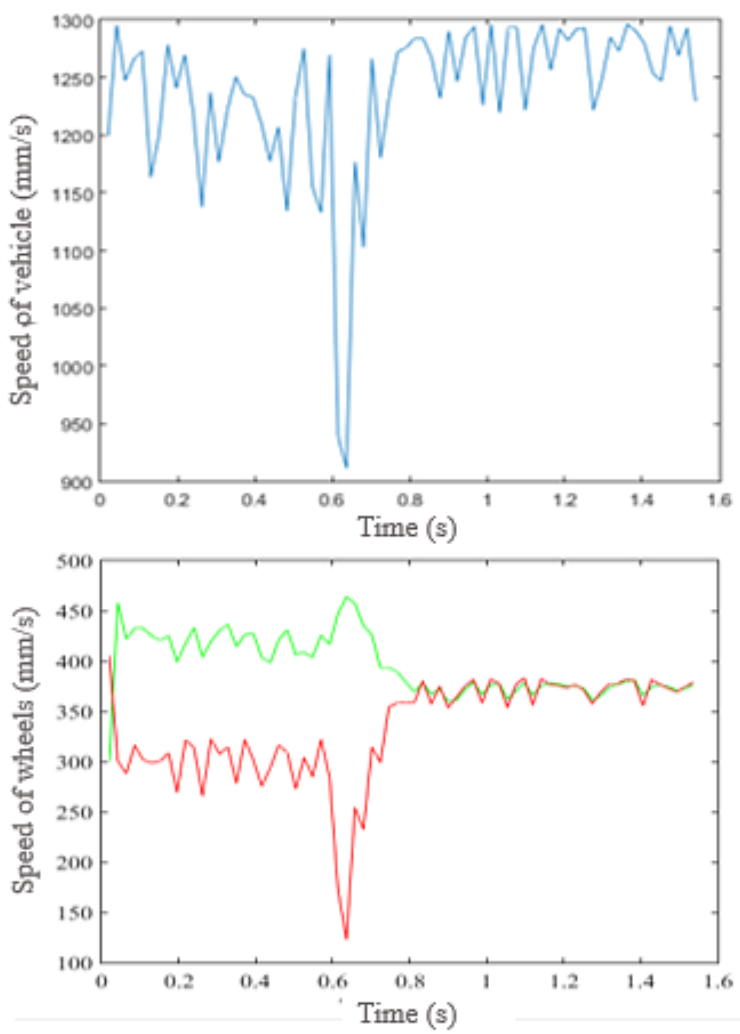

Figure 12. Results of speeds (red: left wheel; green: right wheel) for $\mathrm{ABC}$ section on working map.

Figure 12 decrease to $1.25 \mathrm{~m} / \mathrm{s}$ to maintain the errors not larger than expected. The system keeps driving rule for differential vehicle model such that velocity of right wheel must be greater than left wheel to turn left direction.

The same circumstance on C-D-E section as Figure 13, the greatest errors occur at D point when vehicle is in turn. The

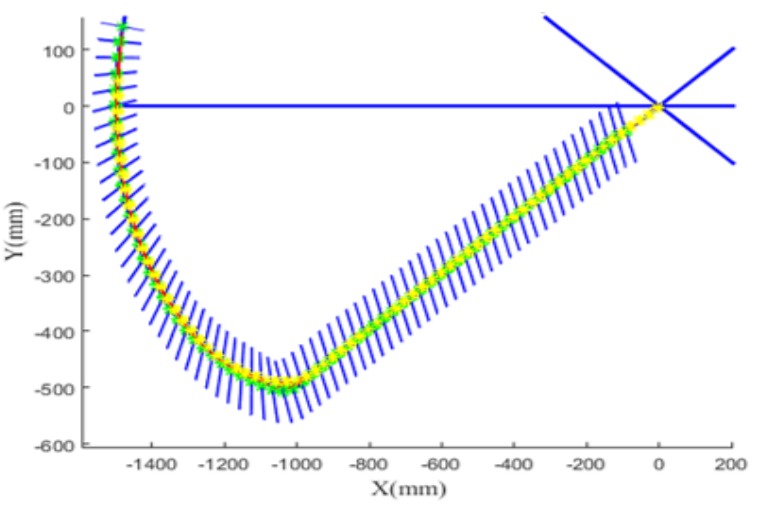

Figure 13. Simulation results for CDE section on working map.
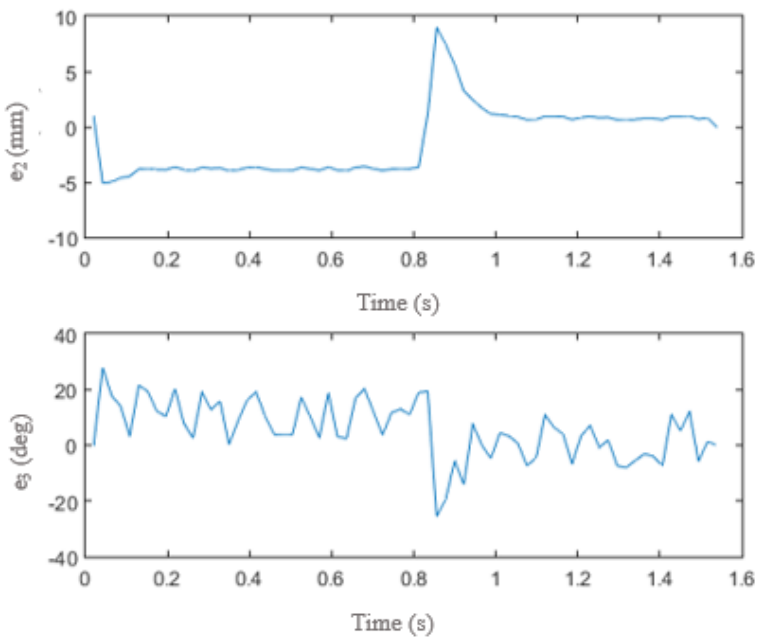

Figure 14. Results of tracking error for CDE section on working map.

maximum $e_{2}$ error is approximately $10 \mathrm{~mm}$ whilst $e_{3}$ is about -23 degree as Figure 14. If vehicle deviates from reference path, the designed controller would drive its back as Figure 15. It takes several times to transpire the calibrated process.

Contrarily, in E-F-C section as Figure 16, vehicle obligates turn right at corner $\mathrm{F}$. Therefore, the $e_{2}$ and $e_{3}$ error are peaked at that time in Figure 17. After that, they alter slightly in bounded condition. During its execution, the system is forced to carry out a decision at point $\mathrm{C}$. The host computer orders its to continue in linear trajectory. It is also confirmed in Figure 18 where speeds of vehicle vary. If the latency time appears in communication, maybe, the worse performance could be made. Finally, autonomous vehicle passes C-G-A section as Figure 19 before it closes a loop. In this case, the following errors in Figure 20 continuously mutate due to driving control scheme. In Figure 21, at each turn, the speeds of wheels are altered to pilot whereas slower velocity of vehicle helps to keep tracking. 

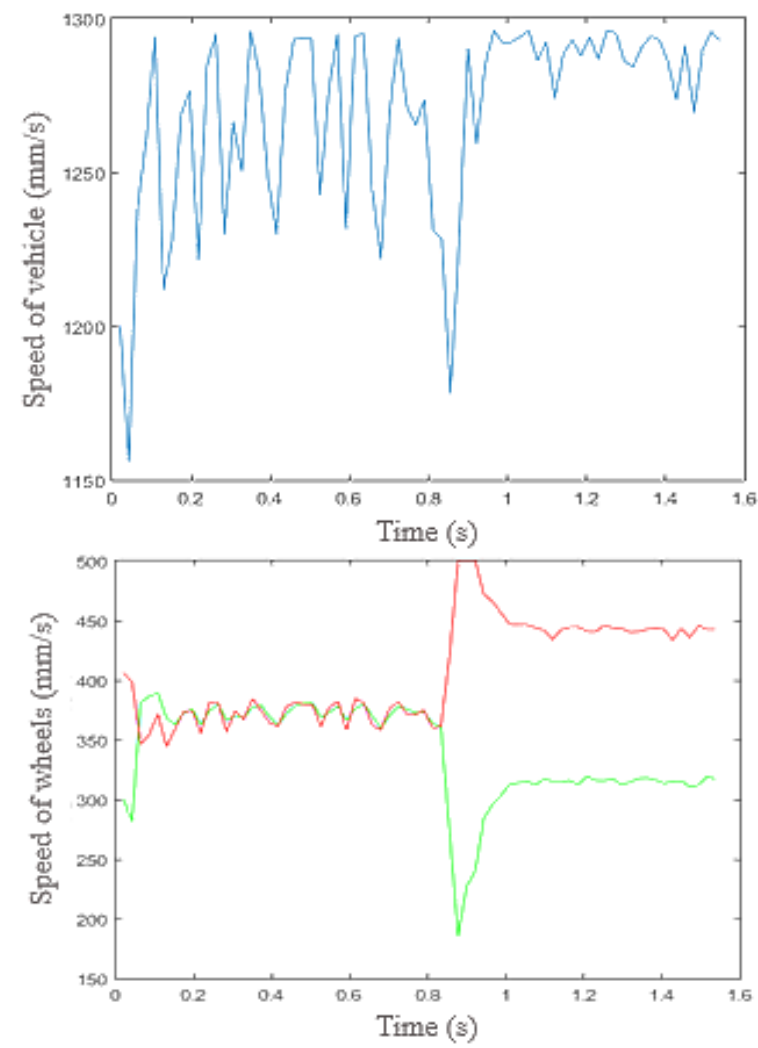

Figure 15. Results of speeds (red: left wheel; green: right wheel) for CDE section on working map.

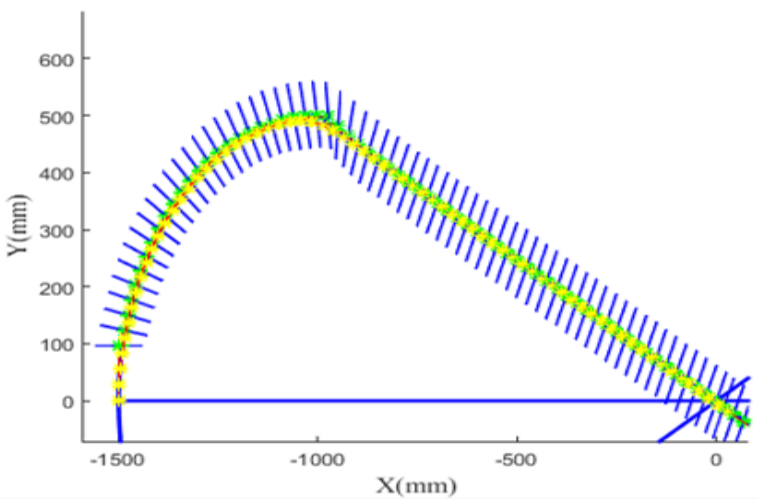

Figure 16. Simulation results for EFC section on working map.

\section{Conclusions}

In this paper, a mechanical architecture for autonomous ground vehicle is introduced to handle multi functions in practical factory. The proposed design is feasible, low cost and complies industrial standards while it still maintains flexible motion. The proposed controller ensures that tracking error converges to zero when time goes to infinity. From the results of research, it could be achievable applications in logistics, manufacturing industry and education.
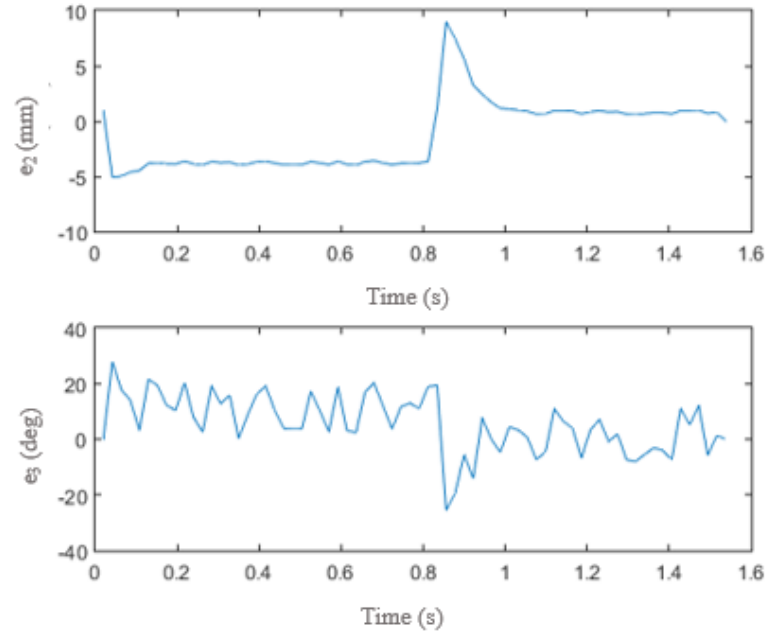

Figure 17. Results of tracking error for EFC section on working map.
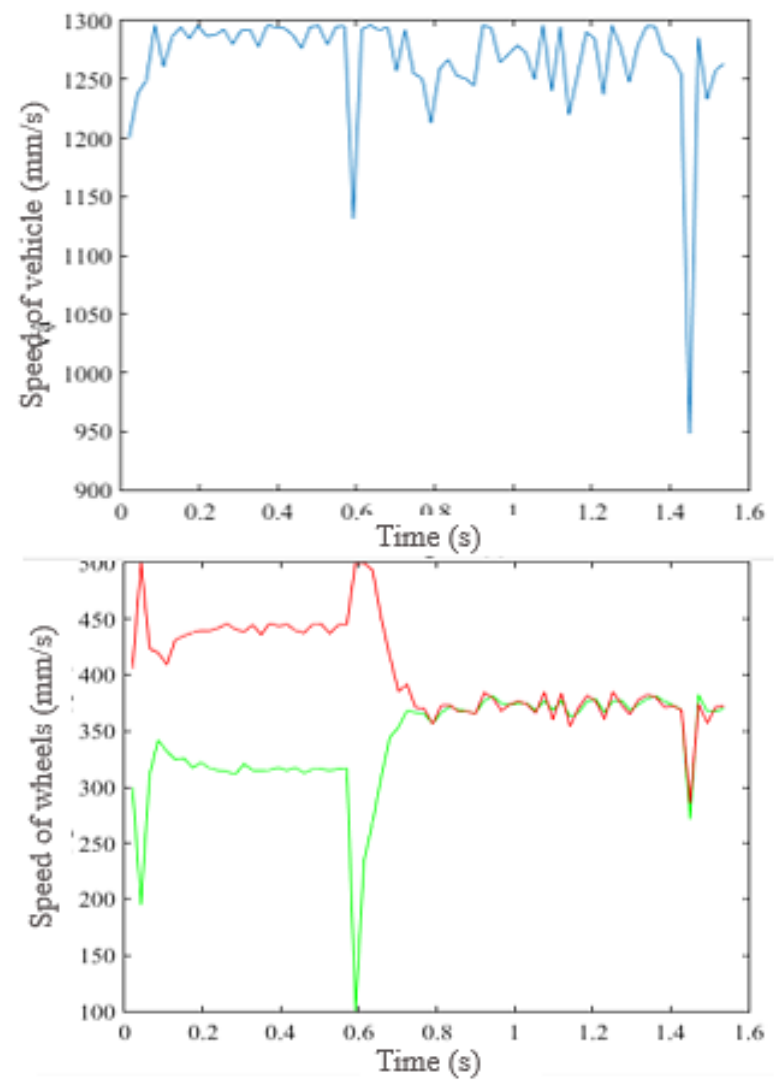

Figure 18. Results of speeds (red: left wheel; green: right wheel) for EFC section on working map.

\section{Conflict of Interest}

No potential conflict of interest relevant to this article was reported. 


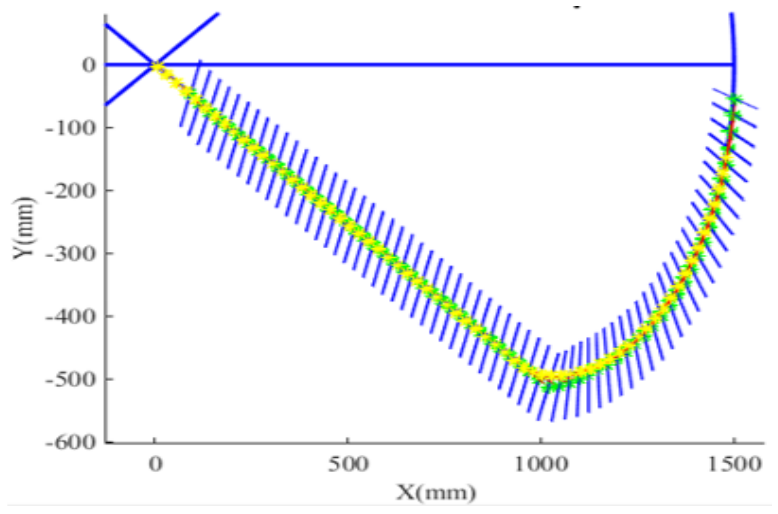

Figure 19. Simulation results for CGA section on working map.
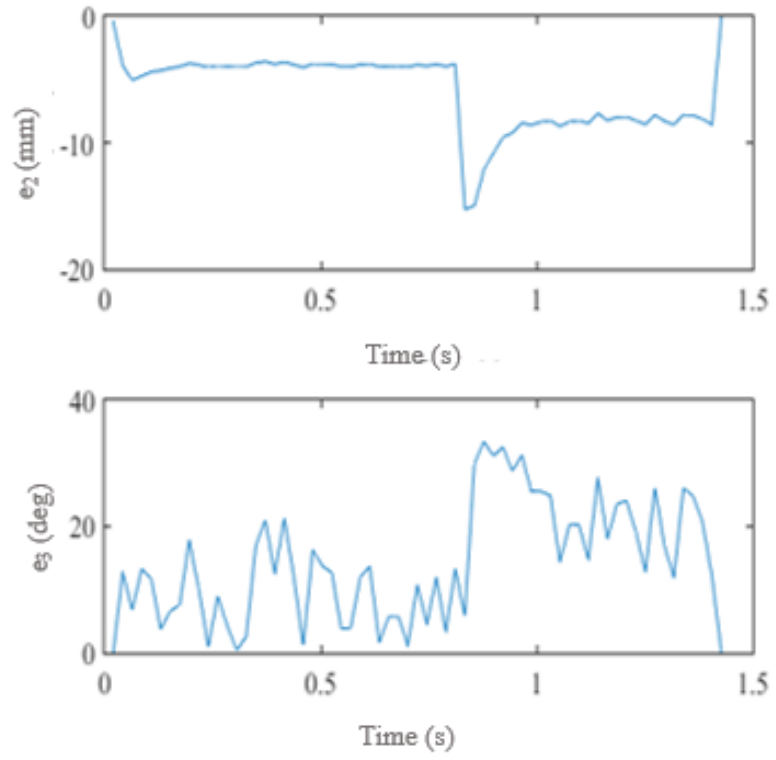

Figure 20. Results of tracking error for CGA section on working map.

\section{Acknowledgment}

This research is funded by Ho Chi Minh City University of Technology, VNU-HCM, under grant number BK-SDH-20191870240.

\section{References}

[1] J. J. Enright and P. R. Wurman, "Optimization and coordinated autonomy in mobile fulfillment systems," in Proceedings of Workshops at the 25th AAAI Conference on Artificial Intelligence, San Francisco, CA, 2011, pp. 33-38.

[2] H. Q. T. Ngo, T. P. Nguyen, and H. Nguyen, "Research and develop of AGV platform for the logistics warehouse
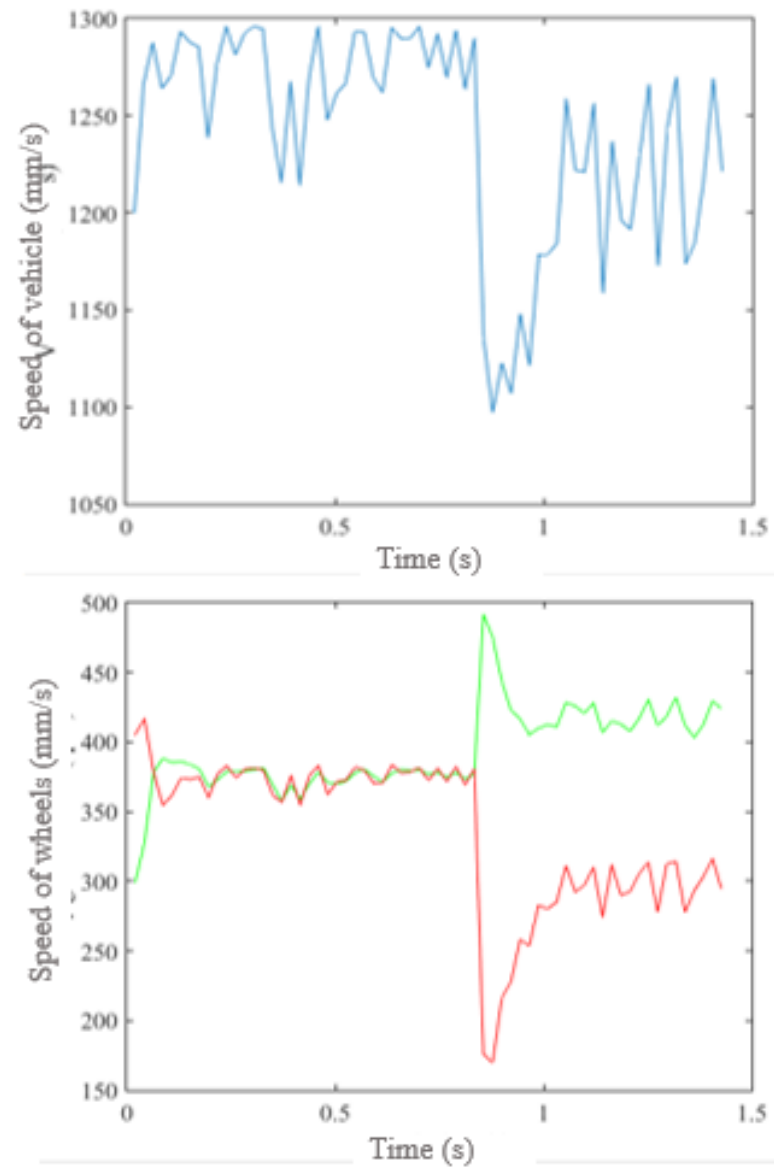

Figure 21. Results of speeds (red: left wheel; green: right wheel) for CGA section on working map.

environment," in Proceedings of the Future Technologies Conference (FTC) 2018. Cham: Springer, 2018, pp. 455465. https://doi.org/10.1007/978-3-030-02683-7_32

[3] H. A. M. Tran, H. Q. T. Ngo, T. P. Nguyen, and H. Nguyen, "Implementation of vision-based autonomous mobile platform to control by A* algorithm," in Proceedings of 2018 2nd International Conference on Recent Advances in Signal Processing, Telecommunications \& Computing (SigTelCom), Ho Chi Minh City, Vietnam, 2018, pp. 39-44. https://doi.org/10.1109/SIGTELCOM.2018.8325802

[4] Q. V. Nguyen, H. M. Eum, J. Lee, and C. H. Hyun, "Vision sensor-based driving algorithm for indoor automatic guided vehicles," International Journal of Fuzzy Logic and Intelligent Systems, vol. 13, no. 2, pp. 140-146, 2013. https://doi.org/10.5391/IJFIS.2013.13.2.140

[5] M. Cheon and H. Lee, "Vision-based vehicle detection system applying hypothesis fitting," International Journal 
of Fuzzy Logic and Intelligent Systems, vol. 17, no. 2, pp. 58-67, 2017. https://doi.org/10.5391/IJFIS.2017.17.2.58

[6] H. Q. T. Ngo and M. H. Phan, "Design of an open platform for multi-disciplinary approach in project-based learning of an EPICS class," Electronics, vol. 8, no. 2, article no. 200, 2019. https://doi.org/10.3390/electronics8020200

[7] M. A. Guney and I. A. Raptis, "A robotic experimental platform for testing and validating warehouse automation algorithms," in Proceedings of 2015 IEEE International Conference on Technologies for Practical Robot Applications (TePRA), Woburn, MA, 2015, pp. 1-6. https://doi.org/10.1109/TePRA.2015.7219693

[8] H. Q. T. Ngo, Q. C. Nguyen, and T. P. Nguyen, "Design and implementation of high performance motion controller for 2-D delta robot," in Proceedings of 2017 7th International Conference on Information Science and Technology (ICIST), Da Nang, Vietnam, 2017, pp. 129134. https://doi.org/10.1109/ICIST.2017.7926505

[9] H. Q. T. Ngo, T. P. Nguyen, and H. Nguyen, "A complete comparison to design complementary filter and Kalman filter for aerial vehicle," International Journal of Mechanical Engineering and Technology, vol. 9, no. 4, pp. 502-513, 2018.

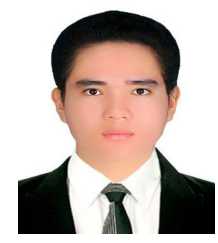

Quang Thinh Truong was born in Vietnam. He received the B.S. degree from Department of Mechatronics Engineering, HCMC University of Technology, VNU-HCM, in 2018. He is currently a graduate student in Department of Mechatronics Engineering, HCMC University of Technology, VNU-HCM. His current research interests include motion control and robotics.

E-mail: 1870240@hcmut.edu.vn

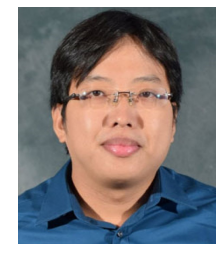

Ha Quang Thinh Ngo is received the B.S. degree from Department of Mechatronics Engineering, HCMC University of Technology (VNU-HCM) in 2006, M.S. and Ph.D. degrees in Department of Intelligent System Engineer- ing from Dong-Eui University, Korea, in 2009 and 2015, respectively. Currently, he is a lecturer in Department of Mechatronics Engineering, HCMC University of Technology, VNU-HCM His research interests consist of motion control, real-time systems and control theory.

E-mail: nhqthinh@hcmut.edu.vn

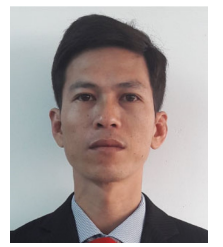

Thanh Phuong Nguyen was born on 1974. He received the Ph.D. degree from Pukyong National University, Korea, in 2008. He is currently an Associate Professor and the Director of HUTECH Institute of Engineering, the Ho Chi Minh City University of Technology, Vietnam. His current research interests include power electronics and robotics.

E-mail: nt.phuong@hutech.edu.vn

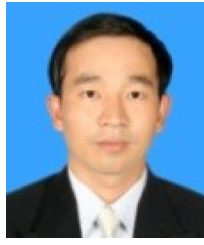

Hung Nguyen was born in Vietnam. He received the B.S. and M.S. degrees from Electrical and Electronics Engineering Department of Ho Chi Minh City University of Technology (HCMUT), Vietnam in 2000 and 2004 respectively. He then received the Ph.D degree from Pukyong National University, Korea, in 2010. He is currently a Associate Professor with HUTECH Institute of Engineering, Ho Chi Minh City University of Technology (HUTECH), Vietnam. His research interests include mobile robot control, automatic guided vehicle, power system control, and renewable energy system.

E-mail: n.hung@ hutech.edu.vn

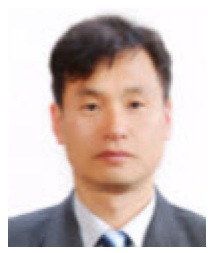

Won-Ho Kim received the B.S., M.S. and $\mathrm{Ph}$.D. degree in School of Electronics Engineering from Kyungpook National University, Daegu, Korea, in 1985, 1988 and 1999, respectively. From 1988 to 1993, he was a researcher of Electronics and Telecommunications Research Institute (ETRI). He is currently a professor in the Major of Robot . Automation Engineering, Dong-Eui University, Busan, Korea. His research interests include robotics and embedded control system.

E-mail: kwh@deu.ac.kr 